\title{
A Relationship Expressed in a Stamp
}

\author{
Proff. Assoc. Dr. llira Sulo (Çaushi) \\ Universita di Tirana \\ Dipartamento di Storia
}

\section{Doi:10.5901/mjss.2013.v4n10p630}

\section{Abstract}

\begin{abstract}
Albania in a historic journey has left numerous evidences in support of our nacional history writting. Those are in content and most variable forms. All together constitute a large reservoir of resources that enables today's articles that we read histori today. The long and beautiful way of the stamp is a valuable asset for all the peoples in the world, in gjeneral and particular for the Albanians. As an adekuate view of the history of the Albanian population, she always had her unrepeatable face. The road that this stamp has followed is similar to the path followed by the Albanian people in history. In this article to explain history I would try to follow somewhat a different path. I will to try to speak through the language of stamps, even for the relations that were created between the two strands of the Adriatic coast. It is in this path, $i$ will try to infer the remaining tracks in the relations that Albania has had with the neighbouring country during the XIX century and onwards.
\end{abstract}

Nel corso del suo viaggio storico, l'Albania ha lasciato tante testimonianze a sostegno della scrittura della storia nazionale. Esse sono varie per quanto riguarda il contenuto e la forma. Tutte insieme costituiscono una fonte ricca di risorse, le quali permettono la scrittura della storia che leggiamo ai giorni d'oggi. Per quanto riguarda la relazione tra Albania e Italia, cerchero' di descrivere una parte della storia di oggi, attraverso un documento differente per quanto riguarda il contenuto e la forma; attraverso quell mezzo che noi siamo abituati a vedere sulle cartoline di auguri o sulle buste di carta, che transmettono messaggi di una differente importanza per la storia. Cercherò di seguire un modo piuttosto originale per spiegare la storia. Sarà proprio la lingua dei francobolli che parlera' riguardo i rapporti che sono state stabilite negli inizi dello stato Albanese attraverso le due coste dell'Adriatico.

II percorso stesso lungo e bello del francobollo e' un bene prezioso per tutti i popoli del mondo in generale, ed in particolar modo per gli Albanesi. Come un quadro adeguato della storia del popolo albanese, essa ha sempre avuto la propria identita' irripetibile. II percorso seguito da questo francobollo, e' molto simile al percorso seguito dalla storia del nostro popolo. Ed e' proprio in questo percorso che cerchero' di dedurre i restanti brani nelle relazioni che l'Albania ha avuto con il paese vicino, l'Italia, nel corso del XIX secolo.

Si riconosce che il francobollo e' nato nel 1840, in Inghilterra ${ }^{1}$. Da quel momento il francobollo ha ridimensionato la propria funzione, in quanto serve non solo come mezzo postale, ma spesso anche come strumento memoriale e come tale può essere connesso ad importanti eventi storici, per ricordare personalità distinte, ecc., ed in questo ridimensionamento si trasforma in un documento importante storico, culturale ed artistico, suscitando cosi' l'interesse anche da studiosi provenienti da ambiti diversi di ricerca.

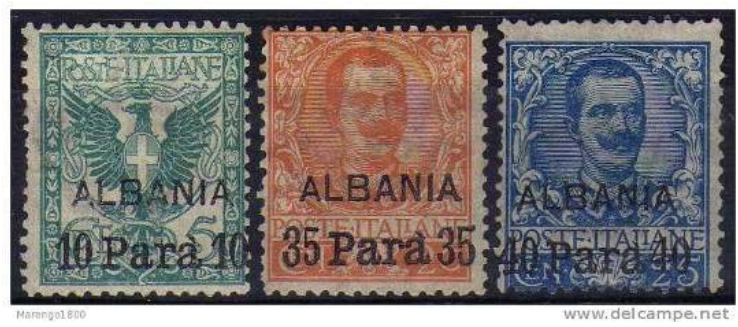

\footnotetext{
1 II primo francobollo è nato in Inghilterra ed è stato lanciato il 6 maggio 1840. Originariamente venne chiamato il "Penny Black" (Penny Black). In poco tempo il "Penny Black" si e' nominato francobollo postale. I primi "Penny Black" riflettevano il ritratto della regina Vittoria, su uno sfondo nero. Successivamente, erano gli Stati Uniti d'America, che hanno iniziato le riprese ad usare i francobolli postali nel 1847, le quali avevano sigillato il ritratto di Benjamin Franklin.
} 
Nel XIX secolo in Albania operavano gli uffici postali (turchi), ancor prima dell'emissione del primo francobollo postale turco. Nel 1840 a Scutari si trovava un ufficio postale (ufficio che usava un sigillo con il n. 1269 (numero romano). Tali uffici sono stati aperti in seguito ad Argirocastro (1866) e Korça (1890). Oltre agli uffici postali turchi che nel 1890-1900 contavano 19 strutture, c'erano uffici postali operanti di origine austriaca, serba, montenegrina, greca e italiana. Sarebbe quest'ultimo l'ufficio che nel 1902 ha emesso tre francobolli con l'inscrizione "Albania"2. Questi francobolli sono stati utilizzati dall'ufficio postale di Scutari, Durazzo e Ioannina. II privilegio che possedeva lo Stato italiano nel dover emettere simili francobolli, si basava sul diritto capitolare funzionante dal periodo dell'Impero Ottomano.

Inizia cosi' una relazione nel campo di produzione di francobolli albanesi, che seguira' in avanti con francobolli contenenti diverse forme e diversi conenuti, a secondo del contesto politico dell'era.

Anche durante gli anni seguenti, saranno rilasciati francobolli messi in circolazione principalmente nelle citta' di Durazzo, Valona e Scutari. Essi saranno in circolazione insieme ai francobolli dell'Impero Ottomano durante gli anni 1902-1910. Come lo stesso francobollo del 1902, anche gli altri francobolli avranno sigillato il ritratto di Vittorio Emanuele III. Essi differivano solo per quanto riguarda la posizione dei ritratti e dai colori. Secondo il commento che fa "Times" del 3 ottobre 1914, si credeva che la circolazione di questi francobolli nei territori appartenenti all'Impero Ottomano, non era solo per ragioni politiche e di concorrenza, ma proveniente anche dal desiderio di guadagnare prestigio di fronte alla popolazione locale.
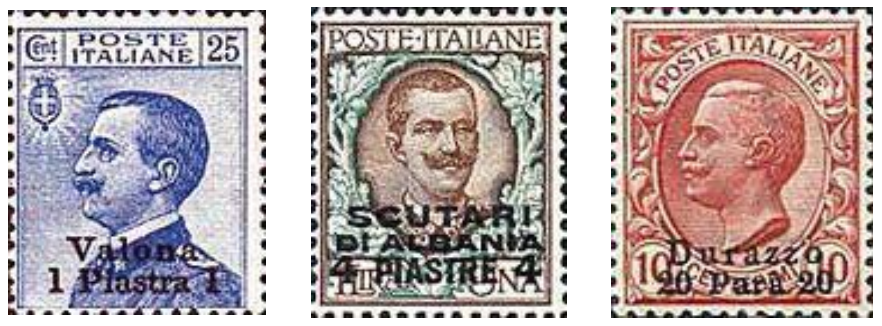

II ritratto dell'Albania sul francobollo ha iniziato a timbrarsi solo dopo la Dichiarazione dell'Indipendenza il 28 Novembre 1912. Appena il 5 dicembre 1912, attraverso un decreto speciale, e' stato stabilito il Servizio Postale e Lef Nosi, un intellettuale noto del tempo, e' stato denominato Ministro delle Poste. Sarà colui che prestera' particolare attenzione all'attività postale in generale ed in particolar modo a quella dell'emissione di francobolli3 .

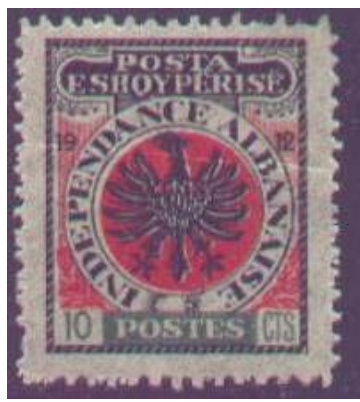

Durante il Governo di Valona si intraprende il primo impegno per il rilascio del francobollo Albanese. II risultato è stato l'emissione di quattro francobolli ufficiali dello Stato Indipendente Albanese. Essi sono stati stampati dalla ditta di S. Modiano a Trieste, Italia 4 . La figura centrale del modello è un'aquila nera sigillata sopra uno sfondo rosso con una cornice a circolo con l'inscrizione in Francese "Indipendance Albanaise" 5 . Nella parte superiore si trova inscritto in bianco su sfondo nero "Posta e Shqypërisë" (La Posta dell'Albania). I francobolli erano recanti l'anno 1912. I timbri sono stati

\footnotetext{
2 K. Mark, Mirdita nella visione filatelica, "Mirdita" Tirana 2004. p.9

3 J. S. Phillips, J. S. Phillips, Francobolli e Poste di Albania ed Epiro 1878-1945, Dhori Koti, Korca, 2002, p .. 83

${ }^{4}$ Si deduce che questo francobollo sia stato stampato nel mese di febbraio 1913

${ }^{5} \mathrm{Si}$ deduce che questa inscrizione in francese rappresenta il desiderio degli Albanesi a declamare l'indipendenza
} 
portati a Valona, ma non sono mai stati usati, a causa dell'aquila avente stampato une testa sola, al contrario dell'ordine Albanese contenente l'aquila con due teste. I francobolli si persero senza traccia nella citta' di Valona.

A causa delle circostanze create al tempo il Governo di Ismail Qemal, ha continuato a mantenere in circolazione francobolli postali del periodo Ottomano. Tale situazione si protrasse per sei mesi, fino a Maggio del 1913, periodo in cui si rilascia e si mette in circolazione il primo francobollo nazionale Albanese (circa 2232 pezzi). La sua realizzazione ha anche una sua storia.

II Governo provvisorio, nel tentativo di esprimere la sua identità anche nel servizio postale, vide Lef Nosi, dopo aver fallito con il francobollo di Trieste, a provare di realizzare un altro francobollo - una marca da bollo - sigillo che poteva essere utilizzata nel servizio postale per quanto riguarda i territori sotto la copertura del Governo di Valona. Era un francobollo - sigillo primitivo, realizzato da un pezzo di gomma, ottenuta da una ruota dove era stata intagliata un aquila a due teste con la scritta Albania, la quale era stata rimossa da un semplice francobollo di bisiness. Con questo modello si sono realizzate diverse prove dove l'aquila era di colore nero e Albania di colore rosso. II ministro raccolse questi campioni per mostrargli ai suoi colleghi.

In principio, il Governo approvo' la proposta, scegliendo il colore nero per di aquila, ma non ha autorizzato l'emissione di francobolli con questa sovrascrizione, a causa del nome mancante del Paese sopra di esso. Questo francobollo - sigillo fatto a mano (primitivo) non e' mai stato emesso.
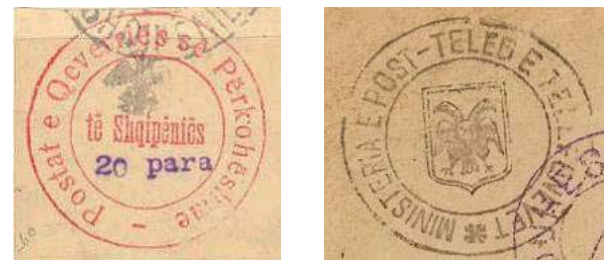

Il primo francobollo - sigillo a mano 6 utilizzato dal governo di Valona, il quale avrebbe dovuto mostrare il pagamento anticipato della spesa di spedizione, è stato ordinato successivamente a Roma. Si costituiva da due cerchi concentrici (di diametro $38 \mathrm{~mm}$ ) con l'inscrizione "Ministria e Post Telegraf Telefonevet" ed in centro uno scudo con l'aquila a due teste. Anche questa marca da bollo era fatta di gomma, solo di colore nero, utilizzata solo su buste contenenti lettere ufficiali, per essere usata in seguito anche su buste private al prezzo di una piastra. II 5 Maggio si riconosce come il primo giorno di uso pubblico di questo francobollo, che duro' fino il 30 Giugno. Ha avuto utilizzo a Berat, Durazzo, Elbasan, Fier, Kavaja, Kruja, Lezha, Lushnje, Peqin, Qukës, Shijak, Skrapar, Tirana e Valona, Scutari omesso. Tale ordine può servire anche a determinare le aree in cui il Governo di Valona esercitava la propria influenza 7 .

Dopo l'utilizzo del primo francobollo - sigillo a mano, si introduce il secondo francobollo, simile al primo $(35 \mathrm{~mm})$, con l'inscrizione "Postat e Qeverries se Perkohshme" (Le Poste del Governo Provvisorio) ed in centro "te Shqipenies" (dell'Albania) ${ }^{8}$. Sono stati realizzati circa 2442 pezzi. Si deduce che le citta' emittenti di questo francobollo sono simili alla prima, aggiungendo la citta' di Scutari. Tutte le buste con tale francobollo provenivano da Valona e si distribuivano nei distretti citati sopra.
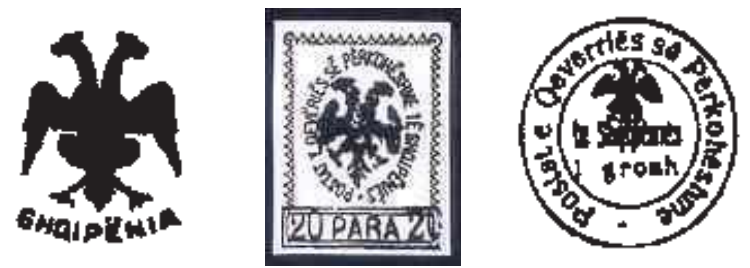

Un altro francobollo che doveva sovramettersi al francobollo circolante nel territorio Albanese, è stata commissionata dal Ministro delle Poste Lef Nosi a Napoli, per mezzo di Ekrem Vlora, l'Albanese Onnipotente accreditato a Roma. Essa

\footnotetext{
${ }^{6}$ Il primo sigillo a mano utilizzato dal governo di Valona maggio 1913.

7 J. S. Phillips, Francobolli e Poste di Albania ed Epiro 1878-1945, Dhori Koti, Korca, 2002, p .. 104

8 Questo sigillo è stato utilizzato anche per "Albanizzare" timbro i francobolli postali ottomani in circolazione. Accadeva spesso al tempo di trovare sulle buste il francobollo ottomano "Albanizzato" ed il secondo francobollo - sigillo.
} 
rappresentava un aquila a due teste, contenente nella parte di basso l'inscrizione Albania. Quest francobollo è stata utilizzato dal Giugno 1913 fino alla fine dello stesso anno.

L'anniversario dell'indipendenza porto' agli impegni di sviluppare un altro tipo di francobollo, collegato a questo evento. Ciò era stato reso necessario dalla mancanza di francobolli a sovrascrizione conteneti l'aquila. Era giunto il momento per l'Albania di avere il proprio francobollo realizzato con la macchina da cucire.

Nonostante gli impegni durante il primo anniversario della dichiarazione dell'indipendenza il 28 novembre 1913, e' stato emesso una serie di 5 francobolli, anche questi realizzati da un sigillo a mano come quello di Ottobre, contenente i tre elementi: l'inscrizione, l'aquila ed il valore nominale. Simultaniamente, e' stato emesso un altro francobollo con guarnizione manuale in metallo, fatto con la dentellatura dalla macchina da cucire. II processo della dentellatura si descrive in modi diversi. Testimonianze del tempo dimostrano che anche per la realizzazione di questo francobollo si cita I'Italia. Secondo Georges Brunel, colui che ha studiato dal 1926 i francobolli albanesi, la macchina da stampa (della dentellatura) è stata ordinata a Trieste 9 .

Questi francobolli si utilizzavano solo a Valona, non essendo distribuiti in altri territori albanesi.

I primi francobolli stampati in modo professionale e ufficialmente rilasciati dal governo albanese sono messi in circolazione il 1 Dicembre 1914. Questi francobolli portano il ritratto del nostro eroe nazionale Skanderbeg. Si tratta del primo francobollo Albanese emesso fino a quel tempo, fatto di dentellatura regolare e dotato di gomma in tutto il foglio.

II ritratto che è stato utilizzato per questo francobollo era stato progettato di utilizzarsi per una medaglia commemorativa, medaglia che non fu mai realizzata. Proprio per questo motivo il Governo Albanese ha ritenuto opportuno utilizzare tale modello per francobolli. II modello è stato realizzato da un noto pittore Ceco (di Praga), Heinz Kautsch, ma venne stampata in una tipografia a Torino, a spese dello Stato Italiano, visto come un dono di un governo ad un altro ${ }^{10}$. Ogni francobollo è stampato in due colori: uno sbiancato che serve come sfondo per le inscrizioni del francobollo.

Si sono emessi sei valori postali in "qindar" e "franchi", (monete del tempo), e la quantità di francobolli stampati era abbastanza elevata per il periodo e per lo standard dell'Albania ${ }^{11}$.
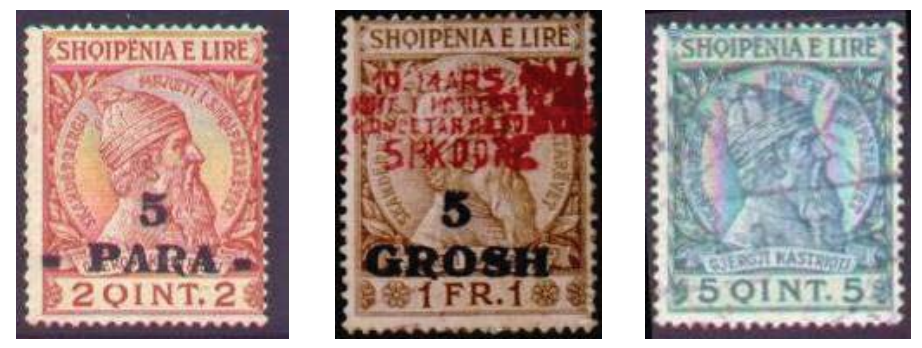

I sei francobolli di questa serie hanno la stessa immagine: il ritratto del nostro eroe nazionale Giorgio Castriota Skanderbeg, ma con colori diversi, secondo I'orientamento della Universal Postal Union (UPU) riguardo l'unificazione dei colori del servizio postale.

L'immagine di Skanderbeg è di profilo, circondato da contorni di un medaglione con la scritta "Skanderbeg Mbreti i Shqiptareve" (Skanderbeg - ॥ Re' degli Albanesi). Nella parte superiore dei francobolli si trova la scritta "Shqipenia e lire" (L'Albania libera). II tiraggio di questi francobolli, come chiaramente documentato dal giornale del tempo "Përlindja e Shqiperise", era molto piu' grande in confronto alle esigenze della circolazione postale. L'intera quantita' è stata di 2.878.500 francobolli. Quindi una percentuale significativa di essi sono stati utilizzati successivamente essendo sovrascritti, per la messa in circolazione di alcuni emmissioni filateliche. Questi francobolli sono stati stampati anche su carta postale. II francobollo e' dotato di tutti i simboli necessari che indicano chiaramente il centro iconico progettato nella forma di un medaglione circondato dalla scritta Skanderbeg, Mbreti i Shqiptareve (Skanderbeg, II Re degli Albanesi) ${ }^{12}$.

\footnotetext{
9 J. S. Phillips, Francobolli e Poste di Albania ed Epiro 1878-1945, Dhori Koti, Korca, 2002, p.119

${ }^{10}$ Parsons, F. P. N. Albania philatelic magazine, tek - J. S. Philipps, cit, p. 121

11500.000 per i francobolli in valore 100, 100.000 per i francobolli in valore di franchi, 150.000 e 100.000 per i francobolli per cartoline semplici del valore 5 cento e 10 cento, 50.000 per cartoline con il pagamento anticipato

12 http://collectionist.tanmarket.com/modules
} 
Successivamente a questo periodo ci troviamo di fronte ad una quiete era nelle relazioni tra i due paesi per quanto riguarda l'emissione di francobolli. Diversi francobolli albanesi con immagini e varie forme continuarono ad essere stampati, non in Italia ma in altri paesi vicini, secondo le congiunture e situazioni politiche.

Nonostante la richiesta per diventare un membro dell'UPU era stata presentata durante il periodo del Governo Di Valona, solo nell'Ottobre del 1922 è stata condotta l'adesione dell'Albania alla UPU.

In solo pochi mesi della sua esistenza, il 7 luglio 1913 il governo provvisorio di Valona ha presentato la richiesta di adesione all'Unione Postale Internazionale, la cui finalizzazione è durata più di otto anni. L'Albania è diventata un membro della UPU solo nel 1922. L'adesione alla UPU fu seguita da vari contratti tra Paesi membri, tra i quali anche I'Italia.

II contratto tra l'Albania e l'Italia e' stato firmato il 6 dicembre 1922, seguito da un periodico dell'UPU del 26 Dicembre 1922, il quale informava gli stati membri di avere un regolare servizio postale tra i due paesi, partendo dal 1 Gennaio 1923 e le poste per e dall'Albania avrebbero circolato attraverso l'Italia.

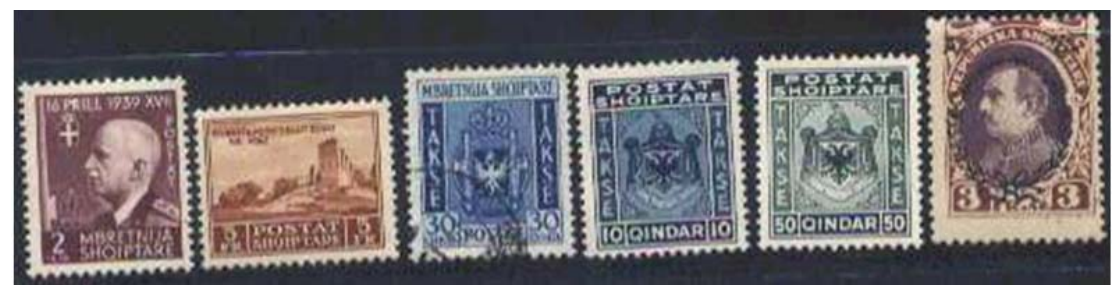

Dal 1 Settembre 1930 tutti i francobolli postali Albanesi furono sostituiti da nuove serie di francobolli stampati a Roma. Proprio in questo periodo si emettono nuovi francobolli appartenenti al Governo di Zog. Essi rappresentano il nuove Re Zog, con un immagine abbastanza affascinante.

Seguendo la serie di questa emissione si trova il francobollo che mostra il ponte sul fiume Mat, un opera connessa con il Re Zog, il quale inizio' la sua costruzione. Un altro francobollo rappresenta le rovine della fortezza dei suoi predecessori, metre la terza immagine riflette il lago di Butrint.

Un grande lavoro ebbe luogo anche per l'emissione dei francobolli contenenti viste aeree. L'intensita' di questa emissione conobbe una crescita con un flusso elevato di voli fra Tirana e Roma (luglio 1931).

All'occasione del 25-immo anniversario della Proclamazione dell'Indipendenza nel 1937 un emissione di francobolli stampati a Roma fu intrapreso. I francobolli furono messi in circolazione il 26 novembre e rimasero validi fino al 28 novembre.

I stampati rispecchiano il cavallo di Scanderbeg, l'immagine dell'acquila che viene proposta di nuovo con una testa solo e con l'inscrizione "Shqipni"13.

Nel 1938, il re Zog sposo' la Principessa... e in questa occasione, simile al anniversario dell'Indipendenza, l'evento storico verra' fissato sul francobollo. Si emettono 9 serie di francobolli con le due figure principali, quella del Re e quella della sua coniuge, con l'inscrizione II Matrimonio del Re, Avenimento Nazionale. I francobolli furono emessi il 25 Aprile e rimasero validi fino al 27 Giugno del 1938.

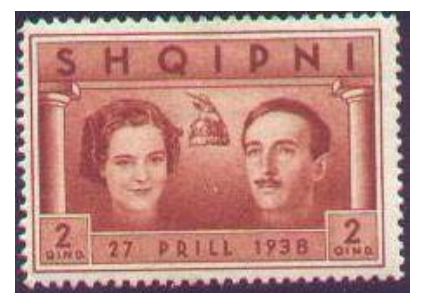

In occasione del decimo anniversario della denominazione di Re Zog, il 1 settembre del 1939, si emettono sette francobolli che promuoverebbero la monarchia. 4 francobolli riflettono l'immagine del Re, francobolli simili a quelli 
dell'inizio del regno di Zog, altri 3 rappresentano un gruppo di emblemi nazionali tra i quali anche l'aquila, questa volta con due teste al centro.
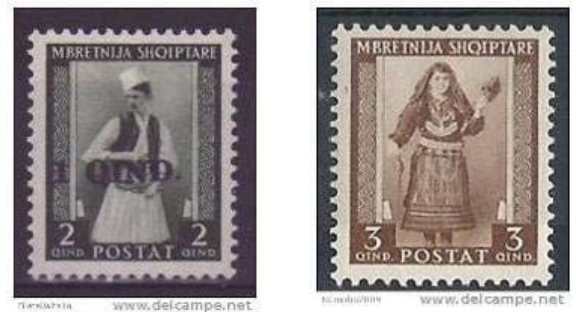

L'emissione dei francobolli varia negli anni 1939-1943. Tutti i francobolli vengono stampati in Italia e il loro aspetto ha una nuova dimensione. Lasciando l'elaborazione di questo periodo ad un altro lavoro di ricerca, vorrei sottolineare che su questi francobolli per la prima volta, oltre ai ritratti che dominavano la situazione politica attuale, si riflettono emissioni fatti di paesaggi Albanesi e monumenti che oggi giorno sono annunciati monumenti di cultura, inoltre, francobolli contenenti immagini di abbigliamenti caratteristici Albanesi del tempo, le quali rappresentano oggi un valore aggiunto al nostro patrimonio culturale.

All'interno di questo breve lavoro, abbiamo cercato di fare un percorso storico, che seguira' con il periodo dopo gli anni 1939. Sono proprio i francobolli che hanno fatto possibile viaggiare negli anni e ci hanno dato l'opportunita' di visualizzare immagini una dopo l'altra nella storia Albanese riguardanti le relazioni con il paese vicino, l'Italia, paese dove si e' stampato in carta una parte della nostra storia.

Forse questo può servire come un esempio perche' nella professione del storicista, qualsiasi documento anche semplice, rappresenta fatti importanti e si puo' leggere ed interpretare in maniera diversa. 